\title{
Effect of Feeding Rice based Distillers Dried Grains with Solubles without or with Different Enzymes on Carcass Characteristics in Broiler Chickens
}

\author{
O. P. Dinani ${ }^{1 *}$, Pramod K. Tyagi ${ }^{2}$, A. B. Mandal ${ }^{3}$, Praveen K. Tyagi ${ }^{4}$ and S. P. Tiwari ${ }^{5}$
}

${ }^{1-4}$ Avian Nutrition and Feed Technology Division ICAR-Central Avian Research Institute Izatnagar, Bareilly, U.P. (243 122), India ${ }^{5}$ Dept. of Animal Nutrition College of Veterinary Science \& A. H. Anjora, Durg, Chhattisgarh (491 001), India

\section{Corresponding Author}

O. P. Dinani

e-mail:dr_dinani@rediffmail.com

\author{
Article History \\ Article ID: AR1887 \\ Received in $15^{\text {th }}$ July, 2018 \\ Received in revised form $29^{\text {th }}$ July, 2018 \\ Accepted in final form $07^{\text {th }}$ August, 2018
}

\begin{abstract}
A biological experiment was conducted to evaluate the effect of feeding rice based distillers dried grains with solubles (rDDGS) as soybean replacement without or with different enzymes on carcass characteristics in broiler chickens. The experiment was conducted as per $3 \times 4$ factorial completely randomized design. A total of 384 broiler chicks of same hatch with uniform weight were used. There were twelve different treatments with 4 replicates for each treatment and each replicate consisted of 8 chicks. Two levels of rice DDGS were taken (12.5 and 15\%). Protease, xylanase and multienzymes supplementation under different treatments were done. The results revealed that no significant $(p>0.05)$ difference was observed due to different levels of rice DDGS and their interaction with different enzymes in carcass traits, cut up parts, visceral organ and immune organ weight except abdomen fat and giblet weight. Abdomen fat was significantly $(p<0.05)$ higher in $15 \%$ rDDGS level with or without xylanase supplementation. Xylanase supplementation increased significantly $(p<0.05)$ giblet weight as compared to multienzymes and without enzyme groups. The study concludes that the feeding of rDDGS levels of $12.5 \%$ and $15 \%$, respectively with or without enzyme supplementation do not have any adverse effects on the carcass traits, but xylanase supplementation improved abdomen fat and giblet weight of broiler chicken at 42 days.
\end{abstract}

Keywords: rDDGS, protease, xylanase, multienzymes, broiler, carcass traits

\section{Introduction}

Poultry production in India has taken a quantum leap in the last four decades. The total poultry population has increased by $12.39 \%$ over the previous census and the total poultry in the country is 729.2 million numbers in 2012 (DAHDF, 2015-16). Feed is the major component in the production of poultry, as it constitutes 65 to $75 \%$ of total production cost. Soybean meal is the major proteinic ingredient used in poultry diet. The estimated requirement for soybean meal will be 8.9 and 11.9 million tons in 2020 and 2025 respectively. However, net deficiency of soybean meal in the country is about 2.5 MMT annually (Mandal, 2017). Due to scarcity of soybean at reasonable price, there is need to utilize locally available alternate protein ingredients. However, only a narrow range of feed ingredients are used due to lack of reliable data on their nutritive quality, feeding value and safe or effective level of inclusion.

Rice tops the list of total cereal production in the country. About $92 \%$ of total rice production is used for human food and about $8 \%$ is used for livestock and poultry feed in form of rice bran, deoiled rice bran, rice polish and broken rice.
Now days, certain newer rice by products are available in appreciable quantities and cheaper rate that can be utilized as protein sources from rice processing industries such as rice based distillers dried grain with solubles (rDDGS).

The DDGS is co-product of the ethanol industry produced during dry milling process. Its availability is increasing due to higher demand for ethanol as biofuel. The DDGS contain all the nutrients from grain in a concentrated form except for the majority of the starch, which has been utilized in the fermentation process during ethanol production. The DDGS contain $65 \%$ distillers grains and $35 \%$ distillers solubles on dry matter basis (AAFCO, 2005). Most of the researches are limited to feeding value of corn, wheat, sorghum, barley based DDGS with very scanty reports on rice DDGS.

On the other hand enzyme supplementations in poultry diets are nutritionally, economically and environmentally justified. It leads to increased feeding value of the dietary raw materials, reduction in the variation of nutrient quality of the diet, increased nutrient digestibility, reduction in water content of the excreta, reduced viscosity of intestinal contents and accelerated rate of passage of digesta through the gastrointestinal tract (Lazaro et al., 2004). However, limited 
information is available on the appropriate enzyme or the combination of enzymes that are specific for broiler chicken ration based on corn-soya diet and SBM partially replaced with rice DDGS.

The carcass traits are closely linked to adequate food and nutrition of broilers. Carcass traits show highly heritability, but there is significant effect of nutrition, protein quality, protein source and amino acid profile of feed ingredients on carcass traits. Nutrient type and composition also affected immune organ weight, internal organ weight and cut up parts (Loar et al., 2010; Harford et al., 2014).

Very scanty research has been done in broiler chickens regarding the effect of feeding rice DDGS on carcass traits without or with different enzymes. In view of the above, a study was conducted to explore effect of feeding rice based DDGS on carcass traits of broiler chickens without or with different enzymes.

\section{Materials and Methods}

\subsection{Experimental design and diets}

A total of 384 CARIBRO Vishal broiler chicks of same hatch and uniform weight were procured from the institutional hatchery and housed in specially designed battery brooder cages with standard watering and feeding facilities. The broiler chicken ration was formulated by using rDDGS as replacement of soybean meal in the basal diets along with enzyme supplementation as per ICAR (2013). The feed ingredients and the nutrient composition of diets as prestarter, starter and finisher as per ICAR (2013) and their analysed values have been given in Table 1, 2 and 3.

The experiment was conducted as per $3 \times 4$ factorial completely randomized design (CRD). A total of 384 broiler chicks of same hatch with uniform weight were used. There were twelve different treatments with 4 replicates for each treatment and each replicate consisted of 8 chicks. Two levels of rice DDGS were taken (12.5 and $15 \%$ ) along with maize soybean based control diet were taken. Protease, xylanase and multienzymes

\begin{tabular}{|c|c|c|c|c|c|c|c|c|c|c|c|c|}
\hline Ingredients & D1 & D2 & D3 & D4 & D5 & D6 & D7 & D8 & D9 & D10 & D11 & D12 \\
\hline Maize & 54.42 & 54.42 & 54.42 & 54.42 & 55.94 & 55.94 & 55.94 & 55.94 & 56.40 & 56.40 & 56.40 & 56.40 \\
\hline SBM & 38.40 & 38.40 & 38.40 & 38.40 & 25.50 & 25.50 & 25.50 & 25.50 & 22.90 & 22.90 & 22.90 & 22.90 \\
\hline DDGS & 0.00 & 0.00 & 0.00 & 0.00 & 12.50 & 12.50 & 12.50 & 12.50 & 15.00 & 15.00 & 15.00 & 15.00 \\
\hline Oil & 3.00 & 3.00 & 3.00 & 3.00 & 1.80 & 1.80 & 1.80 & 1.80 & 1.52 & 1.52 & 1.52 & 1.52 \\
\hline LSP & 1.40 & 1.40 & 1.40 & 1.40 & 1.30 & 1.30 & 1.30 & 1.30 & 1.20 & 1.20 & 1.20 & 1.20 \\
\hline DCP & 1.82 & 1.82 & 1.82 & 1.82 & 1.83 & 1.83 & 1.83 & 1.83 & 1.83 & 1.83 & 1.83 & 1.83 \\
\hline Lysine & 0.00 & 0.00 & 0.00 & 0.00 & 0.23 & 0.23 & 0.23 & 0.23 & 0.27 & 0.27 & 0.27 & 0.27 \\
\hline Methionine & 0.20 & 0.20 & 0.20 & 0.20 & 0.13 & 0.13 & 0.13 & 0.13 & 0.11 & 0.11 & 0.11 & 0.11 \\
\hline Constant $^{*}$ & 0.765 & 0.765 & 0.765 & 0.765 & 0.765 & 0.765 & 0.765 & 0.765 & 0.765 & 0.765 & 0.765 & 0.765 \\
\hline Enzyme & - & + & + & + & - & + & + & + & - & + & + & + \\
\hline Total & 100.01 & 100.01 & 100.01 & 100.01 & 100.00 & 100.00 & 100.00 & 100.00 & 100.00 & 100.00 & 100.00 & 100.00 \\
\hline \multicolumn{13}{|c|}{ Nutrient composition } \\
\hline $\mathrm{CP}$ & 21.99 & 21.99 & 21.99 & 21.99 & 22.01 & 22.01 & 22.01 & 22.01 & 22.02 & 22.02 & 22.02 & 22.02 \\
\hline Lysine & 1.19 & 1.19 & 1.19 & 1.19 & 1.23 & 1.23 & 1.23 & 1.23 & 1.23 & 1.23 & 1.23 & 1.23 \\
\hline Methionine & 0.52 & 0.52 & 0.52 & 0.52 & 0.53 & 0.53 & 0.53 & 0.53 & 0.52 & 0.52 & 0.52 & 0.52 \\
\hline Threonine & 0.83 & 0.83 & 0.83 & 0.83 & 0.82 & 0.82 & 0.82 & 0.82 & 0.82 & 0.82 & 0.82 & 0.82 \\
\hline $\mathrm{Ca}$ & 1.03 & 1.03 & 1.03 & 1.03 & 1.05 & 1.05 & 1.05 & 1.05 & 1.03 & 1.03 & 1.03 & 1.03 \\
\hline$P$ & 0.45 & 0.45 & 0.45 & 0.45 & 0.45 & 0.45 & 0.45 & 0.45 & 0.45 & 0.45 & 0.45 & 0.45 \\
\hline ME $\left(\mathrm{kcal} \mathrm{kg}^{-1}\right)^{* *}$ & 2998 & 2998 & 2998 & 2998 & 2998 & 2998 & 2998 & 2998 & 2999 & 2999 & 2999 & 2999 \\
\hline Cost $\left(₹ \mathrm{~kg}^{-1}\right)$ & 28.52 & 29.03 & 29.13 & 28.93 & 26.36 & 26.86 & 26.96 & 26.76 & 25.86 & 26.37 & 26.47 & 26.27 \\
\hline
\end{tabular}

In prestarter diet *Constant 0.765 includes salt $0.4 \%$; trace mineral premix $0.1 \%$; vitamin premix $0.15 \%$; vit. B complex $0.015 \%$; choline chloride $0.05 \%$ and Toxin binder $0.05 \%$. Trace mineral premix supplied $\mathrm{mg} \mathrm{kg}^{-1}$ diet: $\mathrm{Mn}, 55 ; \mathrm{I}, 1 ; \mathrm{Fe}, 75 ; \mathrm{Zn}$, 60; Cu, 10; Se, 0.15 and Cr, 0.2. The vitamin premix supplied per kg diet: Vit.A, 5000 IU; Vit.D , 2400 IU; Vit.E,15 and Vit.K, 1 mg. Vitamin B complex supplied per kg diet: Vit. $B_{1}, 5$ mg; Vit. $B_{2}, 6$ mg; Vit. B6 5 mg; Vit. 12, $_{15}$ mcg; nicotinic acid, 35 mg; pantothenic acid, $12 \mathrm{mg}$; biotin $0.15 \mathrm{mg}$ and folic acid $0.5 \mathrm{mg}$. Choline chloride supplied per kg diet: choline, $1300 \mathrm{mg}$. (As per ICAR,2013); **calculated value 


\begin{tabular}{lccccccccccccc}
\hline \multicolumn{1}{l}{ Table 2: Ingredients and } & nutrient composition (\%) & of & starter & diets with & or without enzymes for different level of rDDGS \\
\hline Ingredients & D1 & D2 & D3 & D4 & D5 & D6 & D7 & D8 & D9 & D10 & D11 & D12 \\
\hline Maize & 55.63 & 55.63 & 55.63 & 55.63 & 57.66 & 57.66 & 57.66 & 57.66 & 58.10 & 58.10 & 58.10 & 58.10 \\
SBM & 37.10 & 37.10 & 37.10 & 37.10 & 24.10 & 24.10 & 24.10 & 24.10 & 21.40 & 21.40 & 21.40 & 21.40 \\
DDGS & 0.00 & 0.00 & 0.00 & 0.00 & 12.50 & 12.50 & 12.50 & 12.50 & 15.00 & 15.00 & 15.00 & 15.00 \\
Oil & 3.50 & 3.50 & 3.50 & 3.50 & 2.15 & 2.15 & 2.15 & 2.15 & 1.90 & 1.90 & 1.90 & 1.90 \\
LSP & 1.35 & 1.35 & 1.35 & 1.35 & 1.20 & 1.20 & 1.20 & 1.20 & 1.17 & 1.17 & 1.17 & 1.17 \\
DCP & 1.55 & 1.55 & 1.55 & 1.55 & 1.58 & 1.58 & 1.58 & 1.58 & 1.58 & 1.58 & 1.58 & 1.58 \\
Lysine & 0.00 & 0.00 & 0.00 & 0.00 & 0.00 & 0.00 & 0.00 & 0.00 & 0.05 & 0.05 & 0.05 & 0.05 \\
Methionine & 0.10 & 0.10 & 0.10 & 0.10 & 0.04 & 0.04 & 0.04 & 0.04 & 0.03 & 0.03 & 0.03 & 0.03 \\
Constant & 0.765 & 0.765 & 0.765 & 0.765 & 0.765 & 0.765 & 0.765 & 0.765 & 0.765 & 0.765 & 0.765 & 0.765 \\
Enzyme & - & + & + & + & - & + & + & + & - & + & + & + \\
Total & 100.00 & 100.00 & 100.00 & 100.00 & 100.00 & 100.00 & 100.00 & 100.00 & 100.00 & 100.00 & 100.00 & 100.00 \\
Nutrient composition & & & & & & & & & & & \\
\hline CP & 21.52 & 21.52 & 21.52 & 21.52 & 21.54 & 21.54 & 21.54 & 21.54 & 21.50 & 21.50 & 21.50 & 21.50 \\
Lysine & 1.38 & 1.38 & 1.38 & 1.38 & 1.11 & 1.11 & 1.11 & 1.11 & 1.10 & 1.10 & 1.10 & 1.10 \\
Methionine & 0.48 & 0.48 & 0.48 & 0.48 & 0.48 & 0.48 & 0.48 & 0.48 & 0.49 & 0.49 & 0.49 & 0.49 \\
Threonine & 0.78 & 0.78 & 0.79 & 0.79 & 0.81 & 0.80 & 0.80 & 0.81 & 0.81 & 0.81 & 0.81 & 0.81 \\
Ca & 0.95 & 0.95 & 0.95 & 0.95 & 0.96 & 0.96 & 0.96 & 0.96 & 0.96 & 0.96 & 0.96 & 0.96 \\
P & 0.41 & 0.41 & 0.41 & 0.41 & 0.41 & 0.41 & 0.41 & 0.41 & 0.41 & 0.41 & 0.41 & 0.41 \\
ME (kcal kg $\left.{ }^{-1}\right)^{* *}$ & 3050 & 3050 & 3050 & 3050 & 3052 & 3052 & 3052 & 3052 & 3053 & 3053 & 3053 & 3053 \\
Cost (₹ kg ${ }^{-1}$ ) & 28.03 & 28.53 & 28.63 & 28.43 & 25.34 & 25.85 & 25.95 & 25.75 & 24.92 & 25.42 & 25.52 & 25.32 \\
\hline
\end{tabular}

In starter diet *Constant 0.765 includes salt $0.4 \%$; trace mineral premix $0.1 \%$; vitamin premix $0.15 \%$; vit. B complex $0.015 \%$; choline chloride $0.05 \%$ and Toxin binder $0.05 \%$. Trace mineral premix supplied $\mathrm{mg} \mathrm{kg}^{-1}$ diet: $\mathrm{Mn}, 55 ; \mathrm{I}, 1 ; \mathrm{Fe}, 60 ; \mathrm{Zn}, 60$; $\mathrm{Cu}, 10 ; \mathrm{Se}, 0.15$ and $\mathrm{Cr}, 0.2$. The vitamin premix supplied per kg diet: Vit.A, $5000 \mathrm{IU}$; Vit.D, 2400 IU; Vit.E,15 and Vit.K, 1 $\mathrm{mg}$. Vitamin B complex supplied per kg diet: Vit. $B_{1}, 4 \mathrm{mg}$; Vit. $\mathrm{B}_{2}, 6 \mathrm{mg}$; Vit. $\mathrm{B}_{6} 5 \mathrm{mg}$; Vit. $\mathrm{B}_{12}, 15 \mathrm{mcg}$; nicotinic acid, $35 \mathrm{mg}$; pantothenic acid, $10 \mathrm{mg}$; biotin $0.15 \mathrm{mg}$ and folic acid $0.5 \mathrm{mg}$. Choline chloride supplied per kg diet: choline, $1200 \mathrm{mg}$. (As per ICAR, 2013); ** calculated value

supplementation under different treatments were done. The feeding trial was conducted for six weeks and the feed as well as drinking water were provided ad libitum to the birds during the entire experimental period.

\subsection{Sampling and measurement}

All the procedures carried out on the birds were approved by the Institutional Animal Ethics Committee of ICAR-Central Avian Research Institute, Izatnagar (452/01/ab/CPCSEA).

The birds were made to starve for $12 \mathrm{hrs}$ before the actual slaughter, but drinking water was provided ad libitum during starvation period and their body weight were recorded individually before and after starvation. The birds were killed by human method of slaughter.

At the time of slaughter ( $42^{\text {th }}$ day), one male and one female broiler from each replicate group was selected for determination of live weight, dressing, eviscerated yield, blood and feather loss. To attain biometry of the internal organs, the internal organs weight viz giblet (gizzard, heart, liver) and immune organs (spleen, thymus and bursa) were recorded individually after removing the adhering tissue attachments. Weight and length of small intestine (from the duodenum ostium to the ileocecal junction) were recorded. Small intestine length was measured with non stretchable thread and recorded on a graduated scale in $\mathrm{cm}$. Intestinal segment samples was excised and flushed with $0.9 \%$ saline to remove the contents. Organ weights were recorded with the help of a digital balance. The weight: length ratio of the 3 segments was calculated as an indicator of intestinal density. All of the data regarding organ weight and length were expressed per kilogram of BW.

The data collected was subjected to general linear model (GLM) as per factorial CRD to present the results as means and standard errors described by Snedecor and Cochran (1989) by using statistical package for social sciences (SPSS) 16.0 version and the comparison of significant mean differences was as 


\begin{tabular}{lccccccccccccc}
\hline \multicolumn{1}{l}{ Table 3: Ingredients and nutrient composition (\%) of finisher diets for with or without enzymes different level of rDDGS } \\
\hline Ingredients & D1 & D2 & D3 & D4 & D5 & D6 & D7 & D8 & D9 & D10 & D11 & D12 \\
\hline Maize & 62.00 & 62.00 & 62.00 & 62.00 & 64.18 & 64.18 & 64.18 & 64.18 & 64.38 & 64.38 & 64.38 & 64.38 \\
SBM & 31.30 & 31.30 & 31.30 & 31.30 & 18.20 & 18.20 & 18.20 & 18.20 & 15.70 & 15.70 & 15.70 & 15.70 \\
DDGS & 0.00 & 0.00 & 0.00 & 0.00 & 12.50 & 12.50 & 12.50 & 12.50 & 15.00 & 15.00 & 15.00 & 15.00 \\
Oil & 3.22 & 3.22 & 3.22 & 3.22 & 1.80 & 1.80 & 1.80 & 1.80 & 1.60 & 1.60 & 1.60 & 1.60 \\
LSP & 1.20 & 1.20 & 1.20 & 1.20 & 1.00 & 1.00 & 1.00 & 1.00 & 0.96 & 0.96 & 0.96 & 0.96 \\
DCP & 1.45 & 1.45 & 1.45 & 1.45 & 1.50 & 1.50 & 1.50 & 1.50 & 1.50 & 1.50 & 1.50 & 1.50 \\
Lysine & 0.00 & 0.00 & 0.00 & 0.00 & 0.05 & 0.05 & 0.05 & 0.05 & 0.10 & 0.10 & 0.10 & 0.10 \\
Methionine & 0.06 & 0.06 & 0.06 & 0.06 & 0.00 & 0.00 & 0.00 & 0.00 & 0.00 & 0.00 & 0.00 & 0.00 \\
Constant & - & + & + & + & - & + & + & + & - & + & + & + \\
Enzyme & 0.765 & 0.765 & 0.765 & 0.765 & 0.765 & 0.765 & 0.765 & 0.765 & 0.765 & 0.765 & 0.765 & 0.765 \\
Total & 100.00 & 100.00 & 100.00 & 100.00 & 100.00 & 100.00 & 100.00 & 100.00 & 100.00 & 100.00 & 100.00 & 100.00 \\
Nutrient composition & & & & & & & & & & & \\
\hline CP & 19.51 & 19.51 & 19.51 & 19.51 & 19.50 & 19.50 & 19.50 & 19.50 & 19.53 & 19.53 & 19.53 & 19.53 \\
Lysine & 1.20 & 1.20 & 1.20 & 1.20 & 0.98 & 0.98 & 0.98 & 0.98 & 0.98 & 0.98 & 0.98 & 0.98 \\
Methionine & 0.41 & 0.41 & 0.41 & 0.41 & 0.42 & 0.42 & 0.42 & 0.42 & 0.43 & 0.43 & 0.43 & 0.43 \\
Threonine & 0.68 & 0.68 & 0.68 & 0.68 & 0.69 & 0.69 & 0.69 & 0.69 & 0.70 & 0.70 & 0.70 & 0.70 \\
Ca & 0.86 & 0.86 & 0.86 & 0.86 & 0.85 & 0.85 & 0.85 & 0.85 & 0.85 & 0.85 & 0.85 & 0.85 \\
P & 0.38 & 0.38 & 0.38 & 0.38 & 0.38 & 0.38 & 0.38 & 0.38 & 0.38 & 0.38 & 0.38 & 0.38 \\
ME (kcal kg-1 ${ }^{* *}$ & 3100 & 3100 & 3100 & 3100 & 3099 & 3099 & 3099 & 3099 & 3101 & 3101 & 3101 & 3101 \\
Cost (₹ kg ${ }^{-1}$ ) & 26.71 & 27.22 & 27.32 & 27.12 & 24.08 & 24.58 & 24.68 & 24.48 & 23.78 & 24.28 & 24.38 & 24.18 \\
\hline
\end{tabular}

In finisher diet *Constant 0.77 includes salt $0.4 \%$; trace mineral premix $0.1 \%$; vitamin premix $0.15 \%$; vit. B complex $0.015 \%$; choline chloride $0.05 \%$ and Toxin binder $0.05 \%$. Trace mineral premix supplied $\mathrm{mg} \mathrm{kg}^{-1}$ diet: $\mathrm{Mn}, 50 ; \mathrm{I}, 1$; Fe, 50; Zn, 60; $\mathrm{Cu}, 8 ; \mathrm{Se}, 0.15$ and $\mathrm{Cr}, 0.2$. The vitamin premix supplied per kg diet: Vit.A, $5000 \mathrm{IU} ; \mathrm{Vit}^{\mathrm{B}}, 2400$ IU; Vit.E,15 and Vit.K, 0.8 mg. Vitamin B complex supplied per kg diet: Vit. $B_{1}, 4$ mg; Vit. $B_{2}, 6$ mg; Vit. $B_{6} 5$ mg; Vit. $B_{12}, 15$ mcg; nicotinic acid, 30 mg; pantothenic acid, $10 \mathrm{mg}$; biotin $0.15 \mathrm{mg}$ and folic acid $0.5 \mathrm{mg}$. Choline chloride supplied per kg diet: choline, $900 \mathrm{mg}$. (As per ICAR,2013); **alculated value

per Tukey (1949).

\section{Results and Discussion}

The results pertaining to effect of feeding different levels of rDDGS with or without enzymes on carcass traits, organs weight and cut-up parts of the broiler chicken expressed as percentage of live weight are elucidated hereunder.

Effect of feeding different levels of rDDGS on carcass traits with or without enzymes (\% live wt.) is tabulated in Table 4. The results revealed that no significant $(p>0.05)$ difference was observed in dressed weight, eviscerated weight, blood loss, feather loss (\%live wt.), small intestine (S.I.) length, S.I. weight and their density (per kg live wt.). Abdomen fat was significantly $(p<0.05)$ higher in $15 \%$ rDDGS level as compared to $0 \%$ rDDGS, but it did not show any significant ( $p>0.05$ ) difference between 12.5 and $15 \%$ rDDGS levels. Xylanase enzyme supplementation increased significantly $(p<0.01)$ abdomen fat percentage as compared to protease, multienzymes and without enzyme groups. Interaction of rDDGS and enzymes showed significantly $(p<0.01)$ higher abdomen fat content in $0,12.5$ and $15 \%$ rDDGS (with xylanase) groups as compared to control, 0, 12.5 and 15\% rDDGS (with protease) groups.

The results pertaining to effect of feeding different levels of rDDGS on organ weight with or without enzymes (\% live wt.) are presented in Table 5. The results revealed that organ weight in terms of giblet (gizzard, heart and liver) and immune organ weight (thymus, bursa and spleen) did not show any significant $(p>0.05)$ difference as compared to control and other dietary treatments. However, giblet weight was significantly $(p<0.01)$ lower in $15 \%$ rDDGS level as compared to 0 and $12.5 \%$ rDDGS levels. Xylanase enzyme increased significantly $(p<0.05)$ giblet weight as compared to multienzymes and without enzyme groups, but no significant $(p>0.05)$ difference was found between xylanase and protease enzyme in terms of giblet weight. Interaction of rDDGS and 


\begin{tabular}{|c|c|c|c|c|c|c|c|c|c|c|c|}
\hline $\begin{array}{l}\text { Treat- } \\
\text { ment }\end{array}$ & rDDGS\% & Enzyme & Live wt. & $\begin{array}{l}\text { Dress- } \\
\text { ing \% }\end{array}$ & $\begin{array}{l}\text { Eviscer- } \\
\text { ated \% }\end{array}$ & $\begin{array}{l}\text { Blood } \\
\text { loss } \%\end{array}$ & $\begin{array}{c}\text { Feather } \\
\text { loss } \%\end{array}$ & $\begin{array}{l}\text { Abdomen } \\
\text { fat } \%\end{array}$ & $\begin{array}{l}\text { S.I. } \\
\text { length }\end{array}$ & S.I. wt. & $\begin{array}{c}\text { S.I. } \\
\text { density }\end{array}$ \\
\hline $\mathrm{T}_{1}$ & 0 & - & $1689^{b c}$ & 73.0 & 67.9 & 3.1 & 10.6 & $1.28^{\mathrm{ab}}$ & 12.5 & 4.45 & 0.36 \\
\hline$T_{2}$ & 0 & $x$ & $1672^{\mathrm{abc}}$ & 72.9 & 67.7 & 3.1 & 10.6 & $1.54^{\text {cd }}$ & 12.4 & 4.56 & 0.37 \\
\hline$T_{3}$ & 0 & $P$ & $1745^{c}$ & 73.1 & 68.0 & 2.9 & 10.7 & $1.21^{\mathrm{ab}}$ & 11.7 & 4.41 & 0.38 \\
\hline $\mathrm{T}_{4}$ & 0 & $M$ & $1677^{\mathrm{abc}}$ & 72.6 & 67.8 & 3.1 & 10.8 & $1.20^{\mathrm{ab}}$ & 12.5 & 4.50 & 0.36 \\
\hline $\mathrm{T}_{5}$ & 12.5 & - & $1659^{\mathrm{ab}}$ & 73.1 & 68.3 & 2.9 & 10.7 & $1.14^{\mathrm{a}}$ & 12.5 & 4.27 & 0.34 \\
\hline $\mathrm{T}_{6}$ & 12.5 & $x$ & $1715^{b c}$ & 73.5 & 68.0 & 2.8 & 10.6 & $1.51^{c d}$ & 11.7 & 4.22 & 0.36 \\
\hline $\mathrm{T}_{7}$ & 12.5 & $P$ & $1741^{c}$ & 73.1 & 68.2 & 2.7 & 10.6 & $1.32^{\mathrm{abc}}$ & 12.1 & 4.22 & 0.35 \\
\hline $\mathrm{T}_{8}$ & 12.5 & $M$ & $1678^{\mathrm{abc}}$ & 73.4 & 68.2 & 2.7 & 10.7 & $1.52^{\text {cd }}$ & 12.7 & 4.53 & 0.36 \\
\hline $\mathrm{T}_{9}$ & 15 & - & $1612^{\mathrm{a}}$ & 73.5 & 68.6 & 2.9 & 10.7 & $1.39^{\mathrm{bcd}}$ & 12.3 & 4.22 & 0.34 \\
\hline $\mathrm{T}_{10}$ & 15 & $x$ & $1719^{b c}$ & 72.6 & 67.6 & 3.0 & 10.5 & $1.60^{d}$ & 12.2 & 4.34 & 0.36 \\
\hline $\mathrm{T}_{11}$ & 15 & $P$ & $1660^{\mathrm{ab}}$ & 73.4 & 68.2 & 2.8 & 10.6 & $1.26^{\mathrm{ab}}$ & 12.5 & 4.51 & 0.36 \\
\hline \multirow[t]{15}{*}{$\mathrm{T}_{12}$} & 15 & $M$ & $1727^{\mathrm{bc}}$ & 72.6 & 67.7 & 3.0 & 10.6 & $1.35^{\mathrm{abc}}$ & 12.1 & 4.36 & 0.36 \\
\hline & & Pooled SEm \pm & 7.51 & 0.08 & 0.08 & 0.05 & 0.06 & 0.02 & 0.08 & 0.03 & 0.01 \\
\hline & & rDDGS & & & & & & & & & \\
\hline & & 0 & 1696 & 72.9 & 67.8 & 3.0 & 10.7 & $1.30^{\mathrm{a}}$ & 12.3 & 4.5 & 0.37 \\
\hline & & 12.5 & 1699 & 73.3 & 68.2 & 2.8 & 10.7 & $1.40^{\mathrm{ab}}$ & 12.3 & 4.3 & 0.35 \\
\hline & & 15 & 1680 & 73.0 & 68.0 & 2.9 & 10.6 & $1.44^{\mathrm{b}}$ & 12.3 & 4.4 & 0.35 \\
\hline & & Enzyme & & & & & & & & & \\
\hline & & - & $1692^{\mathrm{ab}}$ & 73.2 & 68.3 & 3.0 & 10.7 & $1.30^{\mathrm{a}}$ & 12.4 & 4.31 & 0.35 \\
\hline & & $x$ & $1702^{b}$ & 73.0 & 67.8 & 2.9 & 10.6 & $1.51^{\mathrm{b}}$ & 12.1 & 4.37 & 0.36 \\
\hline & & $\mathrm{P}$ & $1715^{b}$ & 73.2 & 68.1 & 2.8 & 10.7 & $1.27^{\mathrm{a}}$ & 12.1 & 4.38 & 0.36 \\
\hline & & $\mathrm{M}$ & $1656^{a}$ & 72.9 & 67.9 & 2.9 & 10.7 & $1.36^{\mathrm{a}}$ & 12.4 & 4.47 & 0.36 \\
\hline & & Significance & & & & & & & & & \\
\hline & & rDDGS & NS & NS & NS & NS & NS & $p<0.05$ & NS & NS & NS \\
\hline & & Enzyme & $p<0.05$ & NS & NS & NS & NS & $p<0.01$ & NS & NS & NS \\
\hline & & Interaction & $p<0.01$ & NS & NS & NS & NS & $p<0.01$ & NS & NS & NS \\
\hline
\end{tabular}

Values bearing different superscripts within the column differ significantly; NS: Non-significant $(p>0.05)$

enzymes did not show any significant $(p>0.05)$ difference as compared to control and other dietary treatments.

Effect of feeding different levels of rDDGS on cut up parts with or without enzymes (\% live wt.) are tabulated in Table 6. Cut up parts in terms of yield of drumstick, breast, thigh, back, wings and neck did not show any significant $(p>0.05)$ difference as compared to control and other dietary treatments by incorporating different levels of rDDGS $(0,12.5$ and $15 \%)$, enzymes ( $\mathrm{X}, \mathrm{P}$ and $\mathrm{M})$ and their interaction.

Since very scanty references available on rice DDGS feeding on carcass traits. Therefore, some references regarding corn DDGS feeding on carcass traits used here for discussion.

Our results are in agreement with ICAR-CARI (2015), Kim et al. (2016). ICAR- CARI (2015) reported that addition of rDDGS up to $10 \%$ level did not exert any adverse effect on carcass traits in broiler. Kim et al. (2016) reported that finishing broilers (28 to 56 days) can tolerate up to $24 \%$ DDGS in the later phase of production without any detrimental effects on carcass traits. Our results are in disagreement with Wang et al. (2007), Loar et al. (2012); Sonu et al. (2018). Wang et al. (2008) reported carcass yield and breast meat yield reduced when feeding maize DDGS levels greater than $15 \%$ of the diet. Loar et al. (2012) reported dressing percentage and breast meat yield declined in a linear manner with increasing maize DDGS above $14 \%$ level. Sonu et al. (2018) reported that supplementation of multienzymes did not show any significant $(p>0.05)$ difference in carcass traits in the groups fed $15 \%$ and $30 \%$ replacement of soybean meal with DDGS as compared to control in broiler 


\begin{tabular}{|c|c|c|c|c|c|c|c|c|c|c|c|}
\hline $\begin{array}{l}\text { Treat- } \\
\text { ment }\end{array}$ & rDDGS\% & Enzyme & Live wt. & Gizzard & Heart & Liver & Giblet & Spleen & Bursa & Thymus & $\begin{array}{c}\text { S.I. } \\
\text { density }\end{array}$ \\
\hline $\mathrm{T}_{1}$ & 0 & - & $1689^{b c}$ & 2.53 & 0.46 & 2.20 & 5.19 & 0.24 & 0.19 & 0.39 & 0.36 \\
\hline $\mathrm{T}_{2}$ & 0 & $x$ & $1672^{\mathrm{abc}}$ & 2.55 & 0.51 & 2.32 & 5.39 & 0.24 & 0.18 & 0.40 & 0.37 \\
\hline$T_{3}$ & 0 & $P$ & $1745^{c}$ & 2.46 & 0.49 & 2.24 & 5.19 & 0.27 & 0.18 & 0.40 & 0.38 \\
\hline $\mathrm{T}_{4}$ & 0 & $M$ & $1677^{\mathrm{abc}}$ & 2.46 & 0.51 & 2.17 & 5.14 & 0.26 & 0.16 & 0.38 & 0.36 \\
\hline $\mathrm{T}_{5}$ & 12.5 & - & $1659^{\mathrm{ab}}$ & 2.41 & 0.47 & 2.07 & 4.95 & 0.22 & 0.18 & 0.35 & 0.34 \\
\hline $\mathrm{T}_{6}$ & 12.5 & $x$ & $1715^{b c}$ & 2.64 & 0.52 & 2.29 & 5.45 & 0.23 & 0.16 & 0.38 & 0.36 \\
\hline $\mathrm{T}_{7}$ & 12.5 & $P$ & $1741^{c}$ & 2.47 & 0.49 & 2.15 & 5.12 & 0.23 & 0.15 & 0.32 & 0.35 \\
\hline $\mathrm{T}_{8}$ & 12.5 & $\mathrm{M}$ & $1678^{\mathrm{abc}}$ & 2.33 & 0.51 & 2.16 & 5.00 & 0.25 & 0.14 & 0.40 & 0.36 \\
\hline $\mathrm{T}_{9}$ & 15 & - & $1727^{b c}$ & 2.01 & 0.52 & 2.19 & 4.71 & 0.24 & 0.13 & 0.37 & 0.34 \\
\hline $\mathrm{T}_{10}$ & 15 & $x$ & $1719^{\mathrm{bc}}$ & 2.25 & 0.53 & 2.23 & 5.00 & 0.25 & 0.18 & 0.36 & 0.36 \\
\hline $\mathrm{T}_{11}$ & 15 & $P$ & $1660^{\mathrm{ab}}$ & 2.25 & 0.48 & 2.20 & 4.94 & 0.24 & 0.13 & 0.36 & 0.36 \\
\hline \multirow[t]{14}{*}{$\mathrm{T}_{12}$} & 15 & $M$ & $1612^{\mathrm{a}}$ & 2.22 & 0.46 & 2.20 & 4.88 & 0.22 & 0.14 & 0.37 & 0.36 \\
\hline & & $\begin{array}{c}\text { Pooled SEm } \pm \\
\text { rDDGS } \\
\end{array}$ & 7.51 & 0.031 & 0.01 & 0.02 & 0.04 & 0.01 & 0.01 & 0.01 & 0.01 \\
\hline & & 0 & 1696 & $2.50 \mathrm{~b}$ & 0.49 & 2.23 & $5.22^{\mathrm{b}}$ & 0.25 & 0.18 & 0.39 & 0.37 \\
\hline & & 12.5 & 1699 & $2.46 b$ & 0.50 & 2.17 & $5.12^{\mathrm{b}}$ & 0.23 & 0.16 & 0.36 & 0.35 \\
\hline & & 15 & 1680 & $2.18 a$ & 0.50 & 2.20 & $4.88^{\mathrm{a}}$ & 0.24 & 0.15 & 0.37 & 0.35 \\
\hline & & Enzyme & & & & & & & & & \\
\hline & & - & $1692^{\mathrm{ab}}$ & 2.32 & 0.48 & 2.15 & $4.94^{\mathrm{a}}$ & 0.23 & 0.17 & 0.37 & 0.35 \\
\hline & & $x$ & $1702^{b}$ & 2.48 & 0.52 & 2.28 & $5.27^{\mathrm{b}}$ & 0.24 & 0.17 & 0.38 & 0.36 \\
\hline & & $P$ & $1715^{b}$ & 2.40 & 0.49 & 2.20 & $5.08^{a b}$ & 0.25 & 0.15 & 0.36 & 0.36 \\
\hline & & $\mathrm{M}$ & $1656^{a}$ & 2.34 & 0.49 & 2.18 & $5.01^{a}$ & 0.24 & 0.15 & 0.39 & 0.36 \\
\hline & & $\underline{\text { Significance }}$ & & & & & & & & & \\
\hline & & rDDGS & NS & $P<0.01$ & NS & NS & $p<0.01$ & NS & NS & NS & NS \\
\hline & & Enzyme & $P<0.05$ & NS & NS & NS & $p<0.05$ & NS & NS & NS & NS \\
\hline & & Interaction & $P<0.01$ & NS & NS & NS & NS & NS & NS & NS & NS \\
\hline
\end{tabular}

Values bearing different superscripts within the column differ significantly, NS: Non-significant $(p>0.05)$

\begin{tabular}{|c|c|c|c|c|c|c|c|c|c|}
\hline Treatment & rDDGS\% & Enzyme & Live wt. & Drumstick & Breast & Thigh & Back & Wings & Neck \\
\hline $\mathrm{T}_{1}$ & 0 & - & $1689^{b c}$ & 10.2 & 17.3 & 10.0 & 16.4 & 8.9 & 4.5 \\
\hline$T_{2}$ & 0 & $x$ & $1672^{\mathrm{abc}}$ & 10.1 & 16.9 & 10.1 & 16.6 & 8.7 & 4.7 \\
\hline $\mathrm{T}_{3}$ & 0 & $P$ & $1745^{c}$ & 9.9 & 17.4 & 10.5 & 16.6 & 8.8 & 4.4 \\
\hline $\mathrm{T}_{4}$ & 0 & $M$ & $1677^{a b c}$ & 10.6 & 17.4 & 9.7 & 16.9 & 8.8 & 4.7 \\
\hline $\mathrm{T}_{5}$ & 12.5 & - & $1659^{a b}$ & 10.1 & 16.2 & 10.3 & 17.0 & 8.6 & 4.4 \\
\hline $\mathrm{T}_{6}$ & 12.5 & $x$ & $1715^{b c}$ & 10.2 & 17.1 & 10.0 & 17.0 & 8.6 & 4.5 \\
\hline $\mathrm{T}_{7}$ & 12.5 & $P$ & $1741^{c}$ & 10.5 & 17.1 & 10.3 & 17.1 & 8.8 & 4.3 \\
\hline $\mathrm{T}_{8}$ & 12.5 & M & $1678^{a b c}$ & 10.1 & 16.9 & 10.6 & 16.7 & 9.1 & 4.3 \\
\hline $\mathrm{T}_{9}$ & 15 & - & $1727^{b c}$ & 10.2 & 17.3 & 10.6 & 16.8 & 8.7 & 4.4 \\
\hline $\mathrm{T}_{10}$ & 15 & $x$ & $1719^{b c}$ & 10.0 & 17.2 & 10.2 & 16.9 & 8.9 & 4.4 \\
\hline
\end{tabular}




\begin{tabular}{|c|c|c|c|c|c|c|c|c|c|}
\hline Treatment & rDDGS\% & Enzyme & Live wt. & Drumstick & Breast & Thigh & Back & Wings & Neck \\
\hline $\mathrm{T}_{11}$ & 15 & $P$ & $1660^{\mathrm{ab}}$ & 10.2 & 17.3 & 10.5 & 16.3 & 9.0 & 4.6 \\
\hline \multirow{15}{*}{$\mathrm{T}_{12}$} & 15 & $\mathrm{M}$ & $1612^{a}$ & 10.3 & 16.6 & 10.6 & 16.7 & 8.5 & 4.5 \\
\hline & & Pooled SEm \pm & 7.51 & 0.06 & 0.08 & 0.07 & 0.08 & 0.04 & 0.05 \\
\hline & & rDDGS & & & & & & & \\
\hline & & 0 & 1696 & 10.2 & 17.3 & 10.1 & 16.6 & 8.8 & 4.6 \\
\hline & & 12.5 & 1699 & 10.2 & 16.8 & 10.3 & 17.0 & 8.8 & 4.4 \\
\hline & & 15 & 1680 & 10.2 & 17.1 & 10.4 & 16.7 & 8.8 & 4.5 \\
\hline & & Enzyme & & & & & & & \\
\hline & & - & $1692^{\mathrm{ab}}$ & 10.2 & 16.9 & 10.3 & 16.8 & 8.7 & 4.4 \\
\hline & & $x$ & $1702^{b}$ & 10.1 & 17.1 & 10.1 & 16.8 & 8.8 & 4.5 \\
\hline & & $P$ & $1715^{b}$ & 10.2 & 17.3 & 10.4 & 16.7 & 8.9 & 4.4 \\
\hline & & M & $1656^{a}$ & 10.3 & 17.0 & 10.3 & 16.8 & 8.8 & 4.5 \\
\hline & & Significance & & & & & & & \\
\hline & & rDDGS & NS & NS & NS & NS & NS & NS & NS \\
\hline & & Enzyme & $p<0.05$ & NS & NS & NS & NS & NS & NS \\
\hline & & Interaction & $p<0.01$ & NS & NS & NS & NS & NS & NS \\
\hline
\end{tabular}

Values bearing different superscripts within the column differ significantly, NS: Non-significant $(p>0.05)$

chicken. No further references are available in the literature regarding effect of rDDGS feeding on carcass traits of broiler.

\section{Conclusion}

The feeding of rDDGS levels of $12.5 \%$ and $15 \%$, respectively with or without enzyme supplementation do not have any adverse effects on the carcass traits, but xylanase supplementation improved abdomen fat and giblet weight of broiler chicken at 42 days.

\section{Acknowledgement}

Authors are highly thankful to ICAR-Central Avian Research Institute Izatnagar, Bareilly, U.P. 243122 for providing all necessary facilities and inputs.

\section{Conflict of Interest}

Authors declare no conflict of interest.

\section{References}

AAFCO, 2005. Association of American Feed Control Officials. website www.aafco.org.

DAHDF, 2015-16. Department of animal husbandary, dairying and fisheries. Annual report. Government of India, New Delhi.

Harford, I.D., Pavlidis, H.O., Anthony, N.B., 2014. Divergent selection for muscle color in broilers. Poultry Science, 93, 1059-1066.

ICAR, 2013. Nutrient requirements of animals-poultry (ICARNIANP), 13-16.
ICAR- CARI 2015. ICAR-Central Avian Research Institute Annual report, 6.

Lazaro, R., Latorre, M. A., Medel, P., Gracia, M., Mateos, G.G., 2004. Feeding regimen and enzyme supplementation to rye based diets for broilers. Poultry Science 83, 152-160.

Loar, R.E., Moritz, J.S., Donaldson, J.R., Corzo, A., 2010. Effects of feeding distillers dried grains with soluble to broilers from 0 to 28 days post hatch on broiler performance, feed manufacturing efficiency and selected intestinal characteristics. Poultry Science 89, 2242-2250.

Mandal, A.B., 2017. Challenges of feed industries for sustainable poultry production. IPSACON, Lead paper and souvenir, 93-108.

Snedecor, G.W., Cochran, W.G., 1989. Statistical Methods. $7^{\text {th }}$ edn. Oxford and IBH.

Sonu, Sihag, Z.S., Dalal, R., Ahlawat, P., 2018. Effect of multienzyme cocktail on growth performance, digestibility and carcass characteristic of broilers fed soybean meal replaced with distillers dried grain solubles (DDGS) and maize based diet. International Journal of Pure and Applied Biosciences 6(2), 718-724 .

Tukey, J., 1949. Comparing Individual Means in the Analysis of Variance. Biometrics 5(2), 99-114.

Wang, Z., Cerrate, S., Coto, C., Yan, F., Waldroup, P.W., 2007. Effect of rapid and multiple changes in level of distillers dried grain with solubles (DDGS) in broiler diets on performance and carcass characteristics. International Journal of Poultry Science 6, 10, 725-731. 